\title{
Ingestive Behavior
}

National Cancer Institute

\section{Source}

National Cancer Institute. Ingestive Behavior. NCI Thesaurus. Code C16575.

Eating habits and their role in human disease 